January 2, 1996

ITP-SB-96-1

\title{
ACTIONS FOR QCD-LIKE STRINGS
}

\author{
W. Siegel ${ }^{1}$ \\ Institute for Theoretical Physics \\ State University of New York, Stony Brook, NY 11794-3840
}

\begin{abstract}
We introduce a random lattice corresponding to ordinary Feynman diagrams, with $1 / p^{2}$ propagators instead of the Gaussians used in the usual strings. The continuum limit defines a new type of string action with two worldsheet metrics, one Minkowskian and one Euclidean. The propagators correspond to curved lightlike paths with respect to the Minkowskian worldsheet metric. Spacetime dimensionality of four is implied not only as the usual critical dimension of renormalizable quantum field theory, but also from T-duality.
\end{abstract}

1 Internet address: siegel@insti.physics.sunysb.edu. 


\section{INTRODUCTION}

Originally relativistic string theory was introduced to describe hadrons directly. With the advent of quantum chromodynamics the hadronic string was identified as a bound state of gluons and quarks. Unfortunately, the available string models disagreed with several properties expected of QCD strings: (1) They required spacetime dimensions greater than four. This higher dimensionality is usually called the "critical" dimension, and is hidden by eliminating the extra dimensions through compactification or related mechanisms. However, there is no understanding as to why exactly four dimensions remain uncompactified. On the other hand, relativistic quantum field theory of particles (QCD in particular) also has a critical dimension: The condition of perturbative renormalizability requires such theories be defined in no more than four dimensions. In this sense particles, unlike strings, predict the correct dimensionality of spacetime. (An exception might be $\phi^{3}$ theory in six dimensions, but it has an unbounded potential and lacks fermions. A string analog to this pathological theory is the bosonic string in 26 dimensions, which also has a higher critical dimension than better behaved theories.) (2) The underlying partons (gluons and quarks) of hadrons are revealed in processes with large transverse momenta (such as fixed-angle elastic scattering), through the power-law behavior caused by the parton propagators. Unfortunately, the known string theories have Gaussian dependence on these momenta in such limits [1]. (However, Green [2] has found that power-law behavior can be obtained from the usual strings by replacing Neumann boundary conditions with Dirchlet ones [3].) This behavior is closely related to the renormalizability of these string theories in greater than four dimensions.

These and other problems led to the reinterpretation of the known strings as "fundamental" strings, whose perturbative states were not to be identified with hadrons but with gluons and quarks, as well as gravitons, leptons, etc. However, it would still be desirable for strings whose states are identified with the fundamental particles to have critical dimension four. Thus, if it is possible to find a new kind of string theory that is capable of describing hadrons, such a theory might also be preferable for describing fundamental particles. (Of course, there would still be at least the distinction that some fundamental particles are massless, while all hadrons are massive.)

One approach that helps to explain the differences between fundamental strings and composite strings is to replace the worldsheet with a lattice corresponding to a Feynman diagram of an underlying scalar field theory [4]. By identifying the functional integration over worldsheet metrics in the quadratic string action [5] with a 
sum over all lattices [6], and using the large- $\mathrm{N}$ expansion to define surfaces on these lattices [7], a continuum limit can be defined for these lattice models that yields the usual string (at least for the bosonic Liouville string in $\mathrm{D} \leq 1$, but formally for all D) [8]. The key ingredient [4] is to note that the (worldsheet) continuum limit of the product of Gaussian propagators in a Feynman diagram is the exponential of the usual (mechanics) string action. Because Gaussian propagators $\left(e^{-p^{2}}\right)$ are used, (1) all momentum integrations are finite (removing any trace of the renormalizability condition $\mathrm{D}=4$ ), and (2) any parton behavior will give Gaussian behavior rather than power-law. (Usually it is argued that $e^{-p^{2}} \approx 1 /\left(1+p^{2}\right)$, but clearly this approximation cannot describe massless particles such as gluons, and is also inaccurate for large momenta.)

Our approach will be to modify the random lattice formulation of string theory so that the underlying field theory has true $1 / p^{2}$ propagators. In general, we then expect to be able to describe two kinds of strings: (1) In the case where the underlying theory is finite (such as $\mathcal{N}=4$ supersymmetric Yang-Mills theory), we expect a gravity string. Finiteness implies conformal invariance (in the absence of classical mass terms), and even if it is spontaneously broken (generating mass) there is at least a (massless) dilaton as its Goldstone boson. If the conformal graviton is among the string states, it can then eat the dilaton to become the ordinary graviton [9]. (2) In the case where the underlying field theory is asymptotically free (such as QCD), we expect a hadronic string. As usual, the mass scale is generated by dimensional transmutation, and all string states might be massive. (On the other hand, the usual string theories already have a mass scale classically, but also have massless states. However, in the random lattice approach the Liouville mode apparently makes even these states massive.) In this paper the theory we consider explicitly is wrong-sign (massless) $\phi^{4}$ theory in four dimensions, which is asymptotically free. (The wrong sign for the coupling, and thus asymptotic freedom, is a consequence of the random lattice approach.)

The outline of the paper is: (a) In the next section we use Schwinger parameters to exponentiate Feynman diagram integrands without changing the propagators. This produces a random lattice action whose classical extrema for any particular Feynman diagram are its Landau singularities. Individual propagators now satisfy T-duality only in $\mathrm{D}=4$ (and when massless). (b) We then take the continuum limit for $\phi^{4}$ theory. The action has the usual worldsheet metric, appearing in the Virasoro conditions, but which is now Minkowskian, and a second metric that is Euclidean and appears in the $x$ equations. The four-point vertices of the lattice have become the worldsheet lightcones with respect to the Minkowskian metric. (c) We discuss modifications of this 
action that might be useful for more general theories. (d) Finally, we demonstrate that $\mathrm{D}=4$ for the continuum string is implied by either renormalizability (as for the lattice theory) or T-duality.

\section{FEYNMAN DIAGRAMS}

The main reason for introducing Gaussian propagators was to allow exponentiation of the integrand of the second-quantized Feynman diagram, so that it could be identified with a (discretized) first-quantized path integral. The exponentiation of propagators is more commonly performed through the use of Schwinger parameters,

$$
\frac{1}{p^{2}}=\int_{0}^{\infty} d \tau e^{-\tau p^{2}}
$$

(For simplicity, we restrict ourselves to massless particles.) Even in this discretized form, Feynman diagrams are very naturally associated with first-quantized path integrals: To make this analysis, we first write a Feynman diagram for a scalar field theory with nonderivative self-interactions in coordinate space as a Fourier transform:

$$
\int d x_{i}^{\prime} d p_{i j} d \tau_{i j} e^{-\sum_{\langle i j\rangle}\left[\frac{1}{2} \tau_{i j} p_{i j}^{2}+i\left(x_{i}-x_{j}\right) \cdot p_{i j}\right]}
$$

where $i, j$ label vertices (and external endpoints), $\langle i j\rangle$ labels links (propagators), and $d x_{i}^{\prime}$ integrations are over just vertices and not free ends of external lines.

Integration over these $x^{\prime}$ s produces delta functions for momentum conservation

$$
\sum_{j} p_{i j}=0
$$

for the propagator momenta $p_{i j}$. The solution is to replace these momenta with loop (and external) momenta:

$$
p_{i j}=p_{I J}=k_{I}-k_{J}
$$

where $I, J$ label the loops; $p_{I J}$ is now labeled by the two loops on either side of the corresponding propagator, instead of the two vertices at either end. (This parametrization works for planar diagrams. Our arguments generalize straightforwardly for more complicated topologies, but the explicit expressions are messier because source terms for external lines modify the form of the momentum conservation law.) The labelling can be uniquely defined by the $1 / N$ expansion: Each loop is then defined as a quark loop (including broken loops for external lines), associated with the indices of the scalar field matrix (scalar $=$ quark + antiquark); the loop momentum $k_{I}$ can then 
be thought of as the quark momentum. Now external momenta are also expressed as differences, so total momentum conservation is automatic, and there is a momentum "translation invariance". The Feynman integral becomes

$$
\int d k_{I}^{\prime} d \tau_{I J} e^{-\sum_{\langle I J\rangle} \frac{1}{2} \tau_{I J}\left(k_{I}-k_{J}\right)^{2}}
$$

This transformation from coordinate space to (loop-)momentum space is the usual T-duality transformation [10]: Starting from the "first-order" form with auxiliary variable $p_{i j}$, instead of integrating out $p$ we integrated out $x$. These are the same manipulations performed on the usual string (as generalized from the continuum [11] to the random lattice [12]), except there there are no $\tau$ 's. The variable dual to the coordinate $x_{i}$ is the loop momentum $k_{I}$, so the duality transformation is a Fourier transformation as well as a transformation to the dual lattice (vertex $\leftrightarrow$ loop). If we had included an external constant spacetime metric, we would also have seen that, while $x_{i}-x_{j}$ is a contravariant vector, $k_{I}-k_{J}$ is a covariant one (as usual for momenta).

Since we now interpret the exponent of the Feynman integrand as a classical mechanics action, the natural thing to do is vary it to find the classical equations of motion. We first note that the $\tau$ 's are constrained to be positive: We can either check the action at $\tau=0$ to see whether it is an extremum, or make a change of variables such as $\tau=\beta^{2}$ or $e^{\beta}$ to an unconstrained variable. The resulting equations are then (in addition to the momentum conservation law, which we already solved)

$$
\tau_{I J}\left(k_{I}-k_{J}\right)^{2}=0, \quad \sum_{J} \tau_{I J}\left(k_{I}-k_{J}\right)=0
$$

These are exactly the Landau equations for the singularities of S-matrices (as derived from Feynman diagrams or from general properties of S-matrix theory), which correspond to classical configurations of the particles. (The parameters $\tau$ in the Landau equations are often identified with Feynman parameters rather than Schwinger parameters, but our identification is more natural because (1) it is the Schwinger parameters that correspond to the proper time, which is the classical interpretation of the parameters in the Landau equations, (2) these equations follow from the classical interpretation of our discretized action, and (3) Feynman parameters also satisfy some irrelevant algebraic conditions, while the Schwinger parameters are only constrained to be positive, as usual for proper time. The Feynman parameters can be obtained as usual by scaling subsets of the Schwinger parameters and integrating over the scaling parameters, leaving the Feynman parameters as the remaining independent ones.) 
Note that the Landau equations were obtained by variation of the exponent for a single Feynman graph. Since we are interpreting the Feynman diagrams as the first-quantization of a string theory on a random lattice, summation over all graphs replaces integration over the worldsheet metric. We have thus not performed variation with respect to that metric, which is expected to show the stringy properties. (In the usual string theories, it gives the Virasoro conditions, which determine the string spectrum; in our case the interpretation is not yet clear.)

The most important property of this duality transformation for the usual strings is that the dual action is the same as the original action, only on the dual lattice. This is just the statement that the Gaussian propagator takes the same form after Fourier transformation:

$$
e^{-k^{2} / 2} \leftrightarrow e^{-x^{2} / 2}
$$

This is not the case for conventional field theory propagators, even in the massless case, except in four dimensions:

$$
\frac{1}{k^{2}} \leftrightarrow \Gamma\left(\frac{D}{2}-1\right) \frac{1}{x^{D-2}}=\frac{1}{x^{2}} \text { in } \mathrm{D}=4
$$

In terms of the discretized action, this is a consequence of spurious $\tau$ dependence of the measure from $p$ integration and from the required transformation of $\tau$

$$
\tau \leftrightarrow \frac{1}{\tau}
$$

as easily seen from Fourier transformation of a single propagator written in Schwinger parametrized form. Since T-duality is a property of the usual strings, this gives a strong hint that QCD-like strings prefer four dimensions.

\section{CONTINUUM ACTIONS FOR $\Phi^{4}$}

We now look for continuum actions that will exactly reproduce the Feynman diagrams of ordinary field theory when the worldsheet is replaced with a random lattice. Such actions should represent the continuum limit of conventional field theories. The simplest way to derive a continuum action from a random lattice action is to consider a regular square (i.e., flat) lattice, where the continuum limit is easy to take, and covariantize with respect to the worldsheet metric. (This is the analog of the procedure used to couple continuum theories to gravity.) To this continuum action we then add terms depending only on the worldsheet metric, to give the relative weight of different graphs. (Of course, another continuum limit is to replace the propagators 
with one-dimensional worldline actions and sew together the ends, but here we look for two-dimensional continuous worldsheets.) Since $x_{i}-x_{j}$ becomes the worldsheet vector $\partial x, p_{i j}$ becomes a vector (density), so $\tau$ becomes a symmetric tensor (density). For each vertex on a regular square lattice there are two propagators, corresponding to the two independent directions on the worldsheet. This picture generalizes straightforwardly to an arbitrary Feynman diagram in (wrong-sign) $\phi^{4}$ theory. The continuum $\tau$ then has only two components at any point on the worldsheet, so it must be a traceless symmetric tensor.

If we impose the tracelessness condition through a Lagrange multiplier, the continuum action on a curved worldsheet becomes

$$
L_{1}=\frac{1}{2} \tau_{m n}\left(p^{m} \cdot p^{n}-\lambda g^{m n}\right)+i p^{m} \cdot \partial_{m} x+L_{g}\left(g_{m n}\right)
$$

where

$$
L_{g}=\sqrt{-g}(\mu-R \ln N)
$$

contains the usual geometric factors: The cosmological constant $\mu$ on the random lattice gives the coupling constant factor $e^{-\mu}$ for each vertex in the Feynman diagram, while the worldsheet curvature term gives the string coupling $1 / N$ of the topological expansion (at least for its bare value). For this form of the action all the functional integration measures (except possibly that for $g_{m n}$ ) are the usual trivial ones; integrating out $p^{m}$, for example, would introduce nontrivial $\tau$ dependence into the measure. Note that $\tau$ acts as a metric independent from $g$ : On the random lattice $g$ appears implicitly, defining the geometry of the lattice, while $\tau$ appears in addition to that, and is responsible for the $1 / p^{2}$ propagators of the underlying field theory. (In $[10,13]$ $\tau$ was identified with $g$ by approximating it with a nonvanishing constant even at finite order in perturbation theory, resulting in the usual string theory.)

We can also enforce the tracelessness condition through a gauge invariance: By solving the constraint on $\tau_{m n}$,

$$
\begin{gathered}
\tau_{m n}=\hat{\tau}_{m n}-\frac{1}{2} g_{m n} g^{p q} \hat{\tau}_{p q}, \quad \delta \hat{\tau}_{m n}=\zeta g_{m n} \Rightarrow \\
L_{2}=\frac{1}{2} \hat{\tau}_{m n}\left(p^{m} \cdot p^{n}-\frac{1}{2} g^{m n} g_{p q} p^{p} \cdot p^{q}\right)+i p^{m} \cdot \partial_{m} x+L_{g}
\end{gathered}
$$

Furthermore, we can enforce positivity of $\tau_{m n}$ through the redefinition

$$
\begin{gathered}
\hat{\tau}_{m n}=\beta_{m} \beta_{n} \Rightarrow \\
L_{3}=\frac{1}{2} \beta_{m} \beta_{n}\left(p^{m} \cdot p^{n}-\frac{1}{2} g^{m n} g_{p q} p^{p} \cdot p^{q}\right)+i p^{m} \cdot \partial_{m} x+L_{g}
\end{gathered}
$$


(This change of variables also makes the functional integration measure for $\beta$ nontrivial.)

As usual, Weyl scale invariance is broken classically only by the cosmological term, so the naive continuum limit is $\mu \rightarrow 0$. In the usual bosonic string this becomes $\mu \rightarrow$ $\mu_{c}$ when quantum corrections are taken into account, corresponding to a critical value for the $\phi^{4}$ coupling. However, such conclusions might be modified here by dimensional transmutation, since now the Feynman diagrams are not all finite. (On the other hand, in a model corresponding to the Feynman diagrams of a finite supersymmetric Yang-Mills theory, $\mu_{c}=0$ would correspond to the value for S-self-duality.) Also, if we work in the critical dimension (four), we might expect that the string coupling $1 / N$ is unrenormalized. (The continuum limit does not necessarily require $N \rightarrow \infty$ by definition, only the use of an expansion in $1 / N$.)

As for the usual Liouville approach to the random string, the classical equations from varying $g_{m n}$ are not expected to make sense, but need quantum corrections. (Field redefinitions of $g_{m n}$ can cause similar problems for the other fields.) We might consider adding a term

$$
\gamma R \frac{1}{\square} R
$$

to $L_{g}$ to give $g$ nontrivial dynamics, for calculational purposes. The "bare" value of $\gamma$ would then be taken to vanish, while a nonzero coefficient could be generated quantum mechanically through a conformal anomaly.

Since $\tau$ must be positive definite (as the Schwinger parameters were), it is a Euclidean metric. This implies that $g$ must be Minkowskian: If we perform a similarity transformation on $\tau$ to transform it into the identity, then $g$ is traceless, and therefore Minkowskian. (For the usual random lattice and string, $g$ is Euclidean.) The conformal gauge corresponding to the random lattice is then the one where $g$ is off-diagonal, so the constraint on $\tau$ forces it to be diagonal, with the two diagonal components corresponding to the two lightlike directions with respect to $g$. (One could also choose a gauge where $\tau_{m n} \sim \delta_{m n}$. Since not both $g$ and $\tau$ can be fixed, there is always a "spin-2" field.) Thus the edges of the $\phi^{4}$ lattice from which we derived this continuum theory are lightlike paths on a Minkowskian worldsheet; from any vertex the four propagators form the light-cone at that point on the worldsheet. (On the Feynman diagram lattice all curvature is associated with the plaquettes/quark loops, relating to the number of sides, while all vertices and edges/propagators are flat; on the dual lattice, where all plaquettes are squares, all curvature is associated with the vertices.) This is a natural lattice for a Minkowskian metric; e.g., in the case of a regular square 
lattice, the areas $\Delta \sigma^{+} \Delta \sigma^{-}$of the plaquettes are globally Lorentz invariant, being also the $2 \mathrm{D}$ metric.

It should also be possible to generalize this geometric picture to general renormalizable (4D) theories: Since all such couplings are four-point or less, they can all be represented by a subset of the four lightlike worldsheet directions leaving any vertex. This only requires a mechanism to suppress the propagators in some of these directions.

Finally, we can also replace $g$ with a zweibein and use it to flatten the indices on $\tau$ : Since $g$ is Minkowskian, we write $g_{m n}=e_{(m}{ }^{+} e_{n)}{ }^{-}$, and thus

$$
L_{2}^{\prime}=\frac{1}{2} \hat{\tau}_{ \pm \pm} p_{\mp} \cdot p_{\mp}+i p_{ \pm} \cdot e_{\mp}^{m} \partial_{m} x+L_{g}
$$

or

$$
L_{3}^{\prime}=\frac{1}{2}\left(\beta_{ \pm}\right)^{2} p_{\mp} \cdot p_{\mp}+i p_{ \pm} \cdot e_{\mp}^{m} \partial_{m} x+L_{g}
$$

\section{RELATED ACTIONS}

The equations of motion from varying $\hat{\tau}_{m n}$ for $\hat{\tau}_{m n} \neq 0$ are the usual Virasoro conditions with respect to $g_{m n}$ :

$$
p^{m} \cdot p^{n}-\frac{1}{2} g^{m n} g_{p q} p^{p} \cdot p^{q}=0
$$

This is the continuum limit of the Feynman diagram mass-shell condition $p^{2}=0$. If we restrict ourselves to $\phi^{4}$ theory, we thus have $\left(e_{m}{ }^{ \pm} p^{m}\right)^{2}=0$ in the two worldsheet light-cone directions $e_{m}{ }^{ \pm}$, and their opposites $\left(-e_{m}{ }^{ \pm}\right)$, corresponding to the four legs on the four-point vertex. However, for higher-point vertices we would have additional legs, and so require additional world-sheet directions that are linear combinations of $e_{m}{ }^{+}$and $e_{m}{ }^{-}$. This would imply the stronger constraint

$$
p^{m} \cdot p^{n}=0
$$

Unlike the Lagrangian with constrained $\tau_{m n}$, which allows only $\phi^{4}$ or lower-order interactions (since only four worldsheet directions are lightlike), an unconstrained $\tau_{m n}$ would allow vertices with arbitrary powers of $\phi$, as in the usual strings. To guarantee renormalizability, this would require that $\phi$ be dimensionless, so the interaction vertex would require derivatives. However, elimination of the $\lambda g^{m n} \tau_{m n}$ term would (at least classically) completely decouple $g_{m n}$ from the other variables; this might require recoupling through the additional terms representing these vertex factors. Some examples of such theories are 2D nonlinear sigma models and 4D $\mathcal{N}=1$ super Yang-Mills 
theory in superspace. More generally, we have the dimensional analysis for a kinetic term of the form (with $\theta$ counting appropriate to $\mathrm{D}=3,4,6,10$ )

$$
\begin{gathered}
\int d^{D} x d^{2 \mathcal{N}(D-2)} \theta \frac{1}{2} V \square V \\
\Rightarrow \quad 0=-D+\mathcal{N}(D-2)+0+2+0=(\mathcal{N}-1)(D-2) \quad \Rightarrow \quad \mathcal{N}=1 \text { or } \mathrm{D}=2
\end{gathered}
$$

Another possible generalization would be to have $\tau_{m n} g^{m n}$ appear differently from the other components, so it would not act as a Schwinger parameter. For example, if we add a term of the form

\section{$\lambda \bar{\theta} \theta$}

to $L_{1}$, in terms of new anticommuting variables $\theta$ and $\bar{\theta}$, then in the Feynman diagrams integrating over $\lambda, \theta, \bar{\theta}$ and $\tau_{m n} g^{m n}$ produces a momentum-dependent vertex, of the form $\phi^{2}(\partial \phi)^{2}$, since $\tau_{m n} g^{m n}$ multiplies $p^{m}$ 's in two different directions on the worldsheet. In the present case that would lower the critical dimension to two. However, in models with spin this might be a useful method to introduce vertex factors. Also, a term

$$
e^{-\mu} \bar{\theta} \theta
$$

might be an alternative to the cosmological term for introducing the $\phi^{4}$ coupling.

The Lagrangian $L_{1}$ is very similar to one introduced by Polyakov for the usual bosonic string [14]: In first-order form,

$$
L_{P}=\frac{1}{2} \tau_{m n}\left(p^{m} \cdot p^{n}+g g^{m n}\right)+i p^{m} \cdot \partial_{m} x+L_{g}
$$

which differs from $L_{1}$ only by the substitution

$$
\lambda \rightarrow-g
$$

and dropping the positivity condition on $\tau_{m n}$. Varying with respect to the nowLagrange-multiplier $\tau$ gives the Nambu-Goto action; on the other hand, varying with respect to $g_{m n}$ gives

$$
\tau_{m n}=\mu(-g)^{-1 / 2} g_{m n}
$$

which results in the usual quadratic-in- $x$ (and $p$ ) string action (at least classically) with the identification

$$
\mu=\alpha^{\prime}
$$

In the usual random lattice interpretation, both $g$ and $\tau$ are Euclidean in this case. 
This suggests a perturbation expansion for $L_{1}$ based on introducing the length scale through the vacuum value

$$
\left\langle(-g)^{-1} \lambda\right\rangle=\alpha^{\prime-1}
$$

(Such a result might also be expected from one-loop calculations in the usual perturbation expansion, in analogy to the $\mathrm{CP}(\mathrm{n})$ model.) By comparison with the Polyakov action, we see that perturbation about $\langle\lambda\rangle$ would then be perturbation about a conventional string theory, but with the additional variable $\lambda$ (assuming $\lambda$ develops nontrivial dynamics from loops, so it is no longer just a Lagrange multiplier). This would correspond to a different phase from $\phi^{4}$ theory, since in the Polyakov action $g$ and $\tau$ have the same signature (which we would now choose to be Minkowskian in line with our geometric picture for renormalizable field theory). Such an expansion might be suitable for studying Regge behavior.

\section{D=4 FROM THE WORLDSHEET CONTINUUM}

Eliminating $p^{m}$ from $L_{1}$ by functional integration yields

$$
L_{x}=\frac{1}{2}\left(\tau^{-1}\right)^{m n}\left(\partial_{m} x\right) \cdot\left(\partial_{n} x\right)-\lambda \tau_{m n} g^{m n}+L_{g}
$$

and introduces a factor of $\tau^{-D / 2}$ into the measure, where $\tau \equiv \operatorname{det}\left(\tau_{m n}\right)$. On the other hand, eliminating $x$ (and $p^{m}$ ) by solving

$$
\partial_{m} p^{m}=0 \Rightarrow p^{m}=\epsilon^{m n} \partial_{n} k
$$

yields

$$
L_{k}=\frac{1}{2} \tau\left(\tau^{-1}\right)^{m n}\left(\partial_{m} k\right) \cdot\left(\partial_{n} k\right)-\lambda \tau_{m n} g^{m n}+L_{g}
$$

The duality transformation from $x \rightarrow k$ is therefore an invariance of the Lagrangian (classical) if we also transform

$$
\tau_{m n} \rightarrow \tau^{-1} \tau_{m n} \quad\left[=\epsilon_{m p} \epsilon_{n q}\left(\tau^{-1}\right)^{p q}\right], \quad \lambda \rightarrow \tau \lambda
$$

and an invariance of the functional measure (one-loop) if $\mathrm{D}=4$ : Compared to the measure factor $\tau^{-D / 2}$ for $L_{x} \rightarrow \tau^{+D / 2}$ for $L_{k}$, the $\tau_{m n}$ transformation on $\mathcal{D} \tau$ generates an additional factor $\tau^{-2}$ in the measure for $L_{k}$ from the two nonvanishing components of $\tau_{m n}$ (or equivalently, $\tau^{-3}$ from the change in the $\tau_{m n}$ measure together with $\tau^{+1}$ from the change in the $\lambda$ measure). Thus, $\mathrm{D}=4$ arises at one loop as the condition for cancellation of the T-duality anomaly. Note that T-duality is then an invariance of the 
continuum theory; it was not an exact symmetry on the random lattice, since duality replaced each lattice with its dual, which did not appear in the original Feynman diagram summation (at least for $\phi^{4}$ theory). At least for the usual strings, T-duality is related to the standard holomorphic properties, and therefore to conformal invariance.

While variation of these new actions with respect to $\tau_{m n}$ produces the usual Virasoro conditions using the Minkowskian metric $g_{m n}$, variation with respect to $x$ and $p$ results in equations for $x$ similar to the usual except for the replacement of $(-g)^{-1 / 2} g_{m n}$, which has determinant -1 , with the Euclidean $\tau_{m n}$ (a density of the same weight), which has as its determinant a nontrivial scalar. Thus $\tau_{m n}$ acts as the metric for purposes of the $x$ equations of motion. To perform quantum calculations we need to give a vacuum expectation value to $\tau_{m n}$ to define worldsheet propagators for $x$. From 4D dimensional analysis, we expect the expectation

$$
\left\langle\tau_{m n}\right\rangle=\alpha^{\prime} \delta_{m n}
$$

or covariantly

$$
\langle\tau\rangle=\alpha^{\prime 2}
$$

Since we already have

$$
\left\langle g_{m n}\right\rangle=\eta_{m n} \neq \delta_{m n}
$$

this suggests a Higgs effect for 2D general coordinate invariance.

To see the critical dimension following from renormalizability, we perform the continuum version of the calculation used to find the superficial divergence of Feynman diagrams. Including source terms for external momenta (or considering just planar graphs), the functional integral for $L_{k}$ is of the form

$$
\int \mathcal{D}^{2} \tau \mathcal{D}^{D} k^{\prime} e^{-k \tau k}
$$

where all $\sigma$-dependence and indices are implicit ( $\tau$ no longer refers to the determinant), $k$ includes the worldsheet variables $k^{\prime}$ as well as the external (source) momenta $q$, the Lagrange multiplier constraint has been solved, and we have ignored dependence on and integration over $g_{m n}$. Performing the $k^{\prime}$ integration leaves

$$
\int \mathcal{D}^{2} \tau \tau^{-D / 2} e^{-q \tau q}
$$

where $\tau^{-D / 2}$ is the resulting functional determinant (nonlocal in $\sigma$ ). We now consider integration over just the zero-mode of $\tau$ : Writing

$$
\tau_{m n}(\sigma)=\alpha^{\prime} \alpha_{m n}(\sigma)
$$


where $\alpha^{\prime}$ is now a variable independent of the worldsheet coordinates $\sigma$, we have

$$
\int \mathcal{D}^{2} \tau \tau^{-D / 2} d \alpha^{\prime}\left(\alpha^{\prime}\right)^{2-D / 2} e^{-\alpha^{\prime} q \alpha q}
$$

where the $\left(\alpha^{\prime}\right)^{2-D / 2}$ factor in the measure is at every point on the worldsheet (ignoring some finite power of $\alpha^{\prime}$ from the finite number of external lines). The $\alpha^{\prime}$ integration gives the usual $\Gamma$-function factor

$$
\Gamma\left[\left(2-\frac{D}{2}\right) V\right]
$$

where $V$ is the infinite volume of the worldsheet, as measured by the number of points on the worldsheet. More precisely, the usual Feynman diagram integration for $\phi^{4}$ theory gives

$$
\Gamma\left[\left(2-\frac{D}{2}\right) L-\left(2-\frac{E}{2}\right)\right]
$$

where $L$ is the number of loops and $E$ is the number of external lines. For $\mathrm{D}>4$ (nonrenormalizable) it gives $\infty$ (when the argument is an integer), for $\mathrm{D}<4$ (superrenormalizable) the limit $L \rightarrow \infty$ also gives $\infty$, while for $\mathrm{D}=4$ (renormalizable) the result is finite and nonvanishing for $E>4$. Like the duality argument, this suggests that the $4 \mathrm{D}$ theory is better behaved than either $\mathrm{D}>4$ or $\mathrm{D}<4$. In the continuum limit, where $V$ is simply $\infty$, the integration factor is divergent unless $\mathrm{D}=4$ (and sometimes even when $\mathrm{D}=4$, depending on boundary contributions). This shows that the renormalizability condition $\mathrm{D}=4$ for the critical dimension survives the continuum limit.

\section{ACKNOWLEDGMENTS}

I thank Jan de Boer, Gordon Chalmers, Marty Halpern, Martin Roček, Jack Smith, and George Sterman for discussions. This work was supported in part by the National Science Foundation Grant No. PHY 9309888.

\section{REFERENCES}

[1] G. Veneziano, Nuo. Cim. 57A (1968) 190;

V. Alessandrini, D. Amati, and B. Morel, Nuo. Cim. 7A (1971) 797;

D.J. Gross and P.F. Mende, Phys. Lett. 197B (1987) 129, Nucl. Phys. B303 (1988) 407;

D.J. Gross and J.L. Mañes, Nucl. Phys. B326 (1989) 73.

[2] M.B. Green, Phys. Lett. 201B (1988) 42, 266B (1991) 325.

[3] W. Siegel, Nucl. Phys. B109 (1976) 244. 
[4] H.B. Nielsen and P. Olesen, Phys. Lett. 32B (1970) 203;

D.B. Fairlie and H.B. Nielsen, Nucl. Phys. B20 (1970) 637;

B. Sakita and M.A. Virasoro, Phys. Rev. Lett. 24 (1970) 1146.

[5] P.A. Collins and R.W. Tucker, Phys. Lett. 64B (1976) 207;

L. Brink, P. Di Vecchia, and P. Howe, Phys. Lett. 65B (1976) 471;

S. Deser and B. Zumino, Phys. Lett. 65B (1976) 369.

[6] F. David, Nucl. Phys. B257 [FS14] (1985) 543;

V.A. Kazakov, I.K. Kostov and A.A. Migdal, Phys. Lett. 157B (1985) 295.

[7] G. 't Hooft, Nucl. Phys. B72 (1974) 461.

[8] M.R. Douglas and S.H. Shenker, Nucl. Phys. B335 (1990) 635;

D.J. Gross and A.A. Migdal, Phys. Rev. Lett. 64 (1990) 127;

E. Brézin and V.A. Kazakov, Phys. Lett. 236B (1990) 144.

[9] F. Englert, C. Truffin, and R. Gastmans, Nucl. Phys. B117 (1976) 407.

[10] F. David and R. Hong Tuan, Phys. Lett. 158B (1985) 435.

[11] W. Siegel, Phys. Lett. 134 (1984) 318;

T.H. Buscher, Phys. Lett. 194B (1987) 59, 201B (1988) 466.

[12] D.V. Boulatov, V.A. Kazakov, I.K. Kostov, and A.A. Migdal, Nucl. Phys. B275 [FS17] (1986) 641;

W. Siegel, Phys. Lett. 252B (1990) 558.

[13] R. Hong Tuan, Phys. Lett. 173B (1986) 279, 286 (1992) 315, hep-th/9507106 (Orsay preprint).

[14] A.M. Polyakov, Nucl. Phys. B268 (1986) 406. 